\title{
Removal of Multiplicative Gamma Noise from Images via SRAD Model Amelioration
}

\author{
Nacira Diffellah \\ Electronics Department \\ ETA Laboratory \\ Mohamed El Bachir El Ibrahimi University \\ Bordj Bou Arréridj, Algeria \\ diffellahn@gmail.com
}

\author{
Rabah Hamdini \\ Automatics Department \\ SET Laboratory \\ Saad Dahlab University \\ Blida, Algeria \\ hamdinirabah@gmail.com
}

\author{
Tewfik Bekkouche \\ Electromecanics Department \\ ETA Laboratory \\ Mohamed El Bachir El Ibrahimi University \\ Bordj Bou Arréridj, Algeria \\ bekkou66@hotmail.com
}

\begin{abstract}
In this paper, an improved Speckle Reducing Anisotropic Diffusion (SRAD), destined to remove multiplicative gamma noise applied to different images is proposed. The basic idea is to divide the image into several riddled areas and then calculate the Equivalent Number of Look (ENL) of each region. The largest value of the ENL is the best optimal homogeneous region of the image. This optimal choice allows us to solve the major problem of the SRAD algorithm articulated around a visual choice of the homogeneous region which is not satisfactory and causes non-uniformity in this area. To give more validity to the proposed method, several experimentations were conducted using different kinds of images and were approved by some quantitative metrics like PSNR, SNR, VSNR, and SSIM. The computer simulation results confirm the efficiency of the proposed method which outperformances the classical SRAD method.
\end{abstract}

Keywords-multiplicative gamma noise; SRAD; ENL; optimal homogeneous zone; speckle noise

\section{INTRODUCTION}

Images are not immune to contamination by noise that can degrade their visual quality. To deal with this problem, image denoising is the recommended solution. In this context, several restoration filtering algorithms have emerged. These algorithms are based on a variety of denoising techniques [1-8]. The noise in turn is divided into several forms. In this paper, we consider the gamma multiplicative noise. Among the existing techniques, we cite those that are based on anisotropic diffusion. Authors in [1] proposed an algorithm called SRAD, which consists in exploiting the instantaneous coefficient of variation which is a function of local gradient magnitude, and Laplacian operators. However, this method gave good performance in terms of the quality of the restored images, but has the disadvantage of the choice of the homogeneous area determined by a simple subjective visual choice. SRAD [12] needs to know a homogeneous region in the processed image. Although it is not difficult for a user to select a homogeneous region in the image, it is non-trivial for a machine to do that. To remedy this problem an improved method, based on the calculation of the ENL [2], is proposed in this paper, which allows us to analytically determine the optimal homogeneous zone for any image type. Once this area is determined, we apply the SRAD algorithm itself, through simulations carried out on a set of images of different types. The obtained results confirmed the superiority of the proposed over the original SRAD method using several metrics such as: Peak Signal to Noise Ratio (PSNR) [6], Signal to Noise Ratio (SNR) [9], Visual Signal-to-Boise Ratio (VSNR) [10], and Structure Similarity Index Method (SSIM) [13].

\section{THE SPECKLE NOISE MODEL}

Multiplicative noise $[5,13]$ is generally more difficult to remove than additive noise [3]. The model is given by:

$$
f(i, j)=u(i, j) \times \eta(i, j)
$$

where the speckle image $f$ is the product of the original image $u$ and the non-Gaussian noise $\eta$. The indices $i, j$ represent the spatial position over the image. In most applications involving multiplicative noise, the noise content is assumed to be stationary with unitary mean and unknown variance $\sigma^{2}$. The Probability Density Function (PDF) of Erlang (Gamma) noise is given as [4]:

$$
p(x ; K, \theta)=\frac{x^{K-1} e^{-\frac{x}{K}}}{\theta^{k} \Gamma(K)}
$$


For $x>0$ and $K>0, \theta>0$ where $K$ is the number of looks of the image (i.e. the number of independent values averaged) and $\Gamma(\square)$ is the classical Gamma function. $\bar{x}=K \cdot \theta$ is the mean and $\sigma^{2}=K \cdot \theta^{2}$ is the variance. In this work, we assume that the images are corrupted by Gamma distributed noise:

$$
\eta \sim \frac{\eta^{K-1} e^{-\frac{\eta}{K}}}{\theta^{k} \Gamma(K)}
$$

\section{THE SPECKLE REDUCING FAST VARIATIONAL RESTORATION METHOD}

We consider an image $f$ that corresponds to an unknown ground truth image $u$ perturbed with a speckle multiplicative noise $\eta$. The objective is to denoise the available image $f$ and recover an image as close as possible to $u$ according to (1). The SRAD algorithm [1] for the speckle noise reduction in images is summarized as follows:

- Let $u$ be the original image which is normalized on $[0,1]$. Define the smoothing time step and choose the homogeneous region within the original image.

- Compute the speckle scale from the delimited homogenous region which is depended on its variance and mean.

- Compute the Laplacian approximation of the image depending on the nearest-neighbor difference. Compute the nearest-neighbor difference.

- Compute the diffusion coefficient based on the instantaneous coefficient of variation.

- Compute the divergence by which we can update the SRAD function.

\section{THE PROPOSED METHOD}

The ENL is used to evaluate the speckle noise reduction performance of the homogeneous regions in the image. The ENL is defined as:

$$
E N L=\frac{\mu_{u}^{2}}{\partial_{u}^{2}}
$$

where $\mu_{u}$ and $\partial_{n}$ are the estimated mean and standard deviation of the image.

Larger ENL values indicate excellent speckle noise removal ability. ENL is a well-known and effective metric for evaluating the regularization effectiveness on homogeneous areas. The proposed method is summarized in a process of 6 steps:

- Obtain the degraded image $f$ from an imaging system which is the product of the true intensity $u$ and draws from a Gamma distribution.

- Calculate the different evaluation metrics between the original and noisy images.
- Divide the noisy image into several overlapping regions according to the value of $d$. The total number of regions is equal to: $n=(2 d-1)^{2}$, where $d$ is an integer $\neq 0$.

- Calculate the ENL for each region using (4) and choose the greatest value of ENL which corresponds to the most homogeneous region.

- Determine the coordinates of the previous optimal zone which has the greatest value of ENL. Then, apply the SRAD algorithm with the purpose to find the restored image.

- Calculate the metrics between the original image and the restored image and compare the obtained results, before and after restoration.

\section{SIMULATION RESULTS AND DISCUSSION}

In this section, we will compare and discuss the results of the proposed method. The implementation has been tested against a set of images consisting of:

- Image 1: "Image union" [14] with a size of 1024×999 pixels by Kevin Dooley is licensed with CC BY 2.0 [15].

- Image 2: "First NAC Image Obtained in Mercury Orbit" [16] with a size of $1024 \times 1020$ by NASA Goddard Photo and Video, which is also licensed under CC BY 2.0.

- Image 3: "Transportation_airplane_on_lake" [17] with a size of $1289 \times 1024$. This image was acquired from Wikipedia. It was marked as Public Domain or CCO and is free to use.

The images were corrupted with Gamma multiplicative noise having 1 mean and different standard deviation of $4 \%$, $10 \%, 20 \%$, and $60 \%$. In Tables I-III we summarize the simulation results for a sample of given values. Figures 1-3 are composed of:

- The illustration of the position of the homogeneous zone within the noised image, automatically determinated by the ENL metric.

- The number of the homogeneous zone, its coordinates as well as the value of the ENL which is estimated to be the largest by the proposed algorithm.

- The original and the restored images.

- In addition, in order to validate the illustration of these Figures, we also supported the assessment of our approach by all the metrics used.

Tables I-III summarize the results obtained concerning the different metrics used, namely PSNR, SNR, VSNR, and SSIM applied to the original and the noisy images and the real and restored images, by varying each time the number of looks $K=$ 4, $K=10, K=20$, and $K=60$.

In Tables I-III, we clearly notice that the metrics of the proposed method are significantly better than those of the original SRAD method for different noise levels, for the three images used in this paper. 


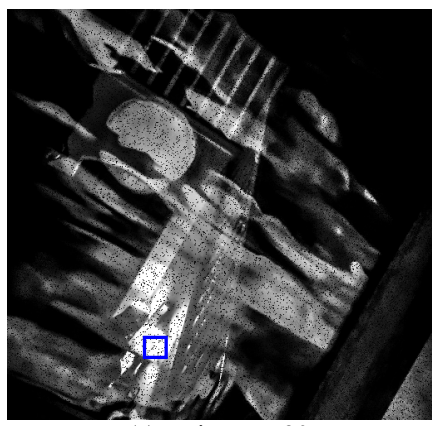

(a) Noisy $K=20$

Region [333.8000 $800.2000 \quad 51.2000 \quad 49.9500], d=20$ PSNR $=21.0963 \mathrm{~dB}, \mathrm{SNR}=9.0602, \mathrm{VSNR}=29.9563 \mathrm{~dB}, \mathrm{SSIM}=0.9514$

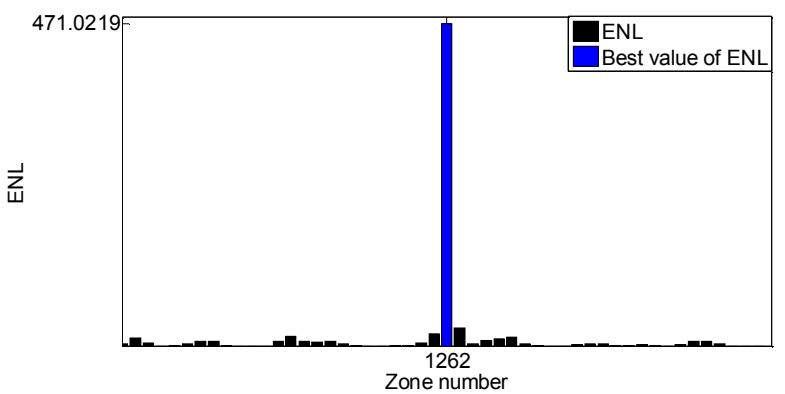

(b) $K=20, \mathrm{ENL}=471.0219$, number $=1262$

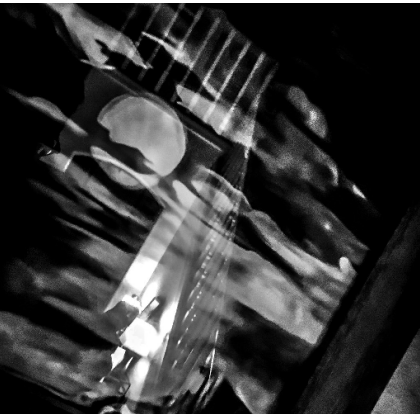

(c) Original

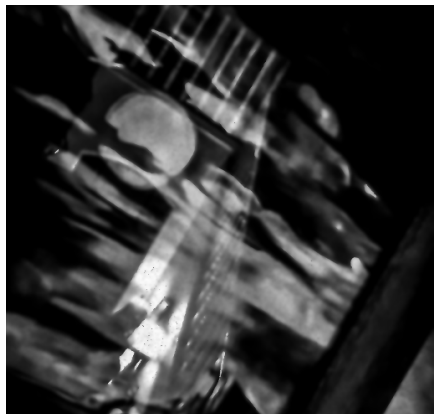

(d) Restored

$\mathrm{PSNR}=26.5126 \mathrm{~dB}, \mathrm{SNR}=13.7435$ $\mathrm{VSNR}=30.8696 \mathrm{~dB}, \mathrm{SIM}=0.9470$

Fig. 1. Results of image 1.

TABLE I. PSNR, SNR, VSNR, AND SSIM OF NOISY AND DESPECKLED IMAGE 1 WITH DIFFERENT LEVELS OF $K$

\begin{tabular}{|c|c|c|c|c|}
\hline & PSNR & SNR & VSNR & SSIM \\
\hline $\boldsymbol{K}=\mathbf{4}$ & 15.1109 & 3.5008 & 26.4856 & 0.8231 \\
\hline Proposed & 20.7247 & 7.3498 & 26.1100 & 0.8760 \\
\hline SRAD & 18.8231 & 4.7549 & 26.1531 & 0.8270 \\
\hline $\boldsymbol{K}=\mathbf{1 0}$ & 17.6972 & 5.8270 & 27.9382 & 0.9040 \\
\hline Proposed & 24.1607 & 11.3095 & 26.4329 & 0.9271 \\
\hline SRAD & 23.5171 & 10.5614 & 26.0267 & 0.9195 \\
\hline $\boldsymbol{K}=\mathbf{2 0}$ & 21.0963 & 9.0602 & 29.9563 & 0.9514 \\
\hline Proposed & 26.5126 & 13.7435 & 30.8696 & 0.9470 \\
\hline SRAD & 23.5093 & 10.5496 & 29.9626 & 0.9193 \\
\hline $\boldsymbol{K}=\mathbf{6 0}$ & 32.8812 & 20.7208 & 33.5205 & 0.9955 \\
\hline Proposed & 28.4062 & 15.9718 & 28.3802 & 0.9643 \\
\hline SRAD & 26.4717 & 13.7056 & 26.0019 & 0.9464 \\
\hline
\end{tabular}

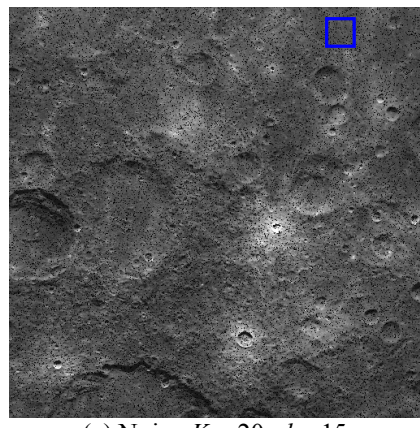

(a) Noisy $K=20, d=15$

Region $\left[\begin{array}{llll}786.0667 & 35.0000 & 68.2667 & 68.0000\end{array}\right]$

$\mathrm{PSNR}=20.0851 \mathrm{~dB}, \mathrm{SNR}=0.4349-, \mathrm{VSNR}=10.5243 \mathrm{~d} 8, \mathrm{SSIM} 0=0.8115$

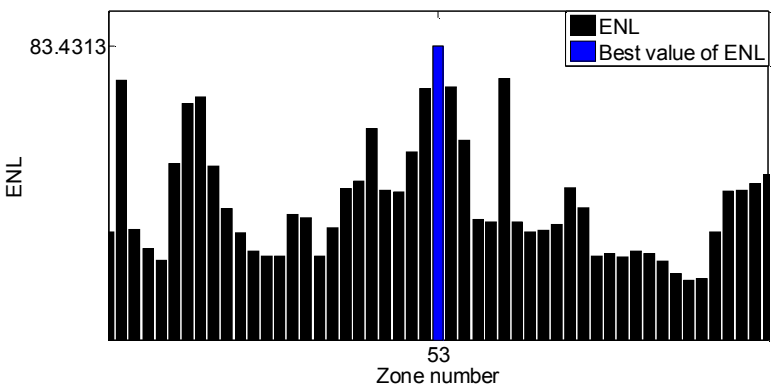

(b) $\mathrm{ENL}=83.4313$, number $=53$

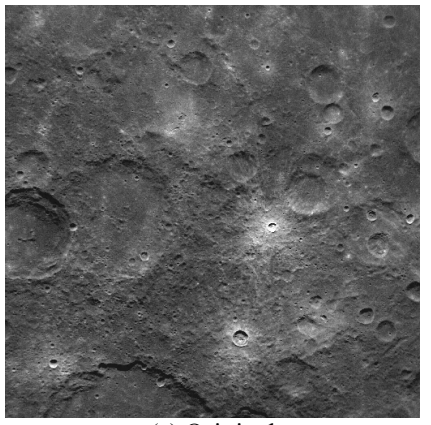

(c) Original

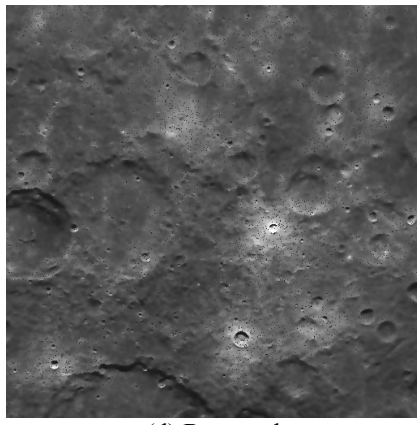

(d) Restored

$\mathrm{PSNR}=24.9378 \mathrm{~dB}, \mathrm{SNR}=3.5459$ $\mathrm{VSNR}=12.4653, \mathrm{SSIM}=0.8247$
Fig. 2. Results of image 2 .

TABLE II. PSNR, SNR, VSNR, AND SSIM OF NOISY AND DESPECKLED IMAGE 2 WITH DIFFERENT LEVELS OF $K$

\begin{tabular}{|c|c|c|c|c|}
\hline & PSNR & SNR & VSNR & SSIM \\
\hline $\boldsymbol{K}=\mathbf{4}$ & 13.9953 & -4.5188 & 5.7907 & 0.6204 \\
\hline Proposed & 19.5193 & 1.2013 & 11.9192 & 0.7196 \\
\hline SRAD & 16.7190 & -0.0049 & 11.5444 & 0.6345 \\
\hline $\boldsymbol{K}=\mathbf{1 0}$ & 16.6044 & -2.6240 & 7.5992 & 0.7688 \\
\hline Proposed & 22.9478 & 2.6145 & 12.3695 & 0.7944 \\
\hline SRAD & 19.0560 & 0.9626 & 11.8564 & 0.7070 \\
\hline $\boldsymbol{K}=\mathbf{2 0}$ & 20.0851 & 0.4349 & 10.5243 & 0.8115 \\
\hline Proposed & 24.9378 & 3.5459 & 12.4653 & 0.8247 \\
\hline SRAD & 21.3566 & 1.9513 & 12.1517 & 0.7625 \\
\hline $\boldsymbol{K}=60$ & 31.7254 & 11.7804 & 19.5227 & 0.9907 \\
\hline Proposed & 27.3115 & 5.9811 & 14.1275 & 0.8929 \\
\hline SRAD & 24.8388 & 3.3729 & 12.2072 & 0.8147 \\
\hline
\end{tabular}




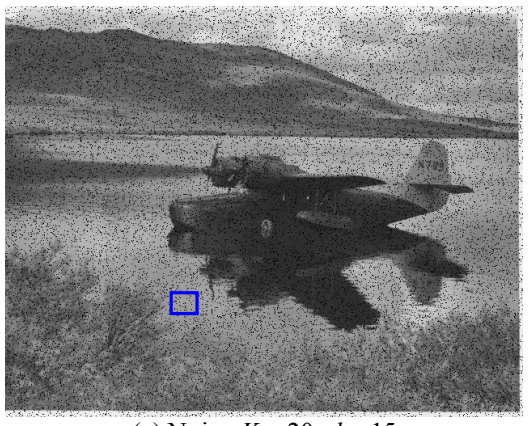

(a) Noisy $K=20, d=15$

Region [419.9250 $717.8000 \quad 64.4500 \quad 51.2000]$ $\mathrm{PSNR}=16.5447 \mathrm{~dB}, \mathrm{SNR}=2.3706, \mathrm{VSNR}=12.1235 \mathrm{~d} 8, \mathrm{SSIM} 0=0.72320$

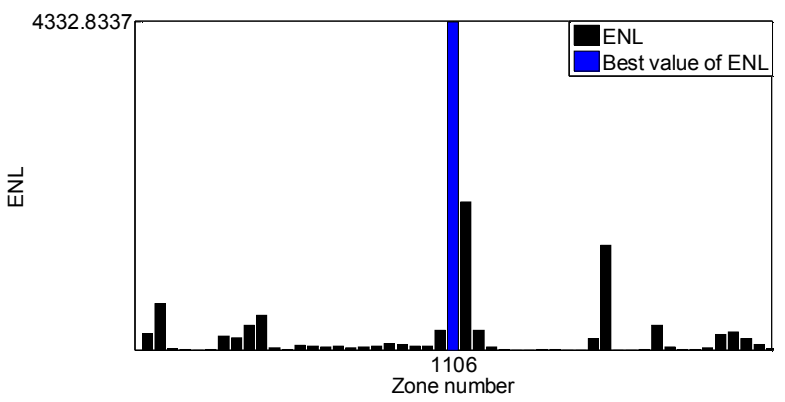

(b) $\mathrm{ENL}=4332.8337$, number $=1106$

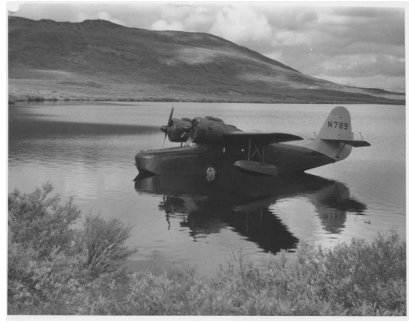

(c) Original

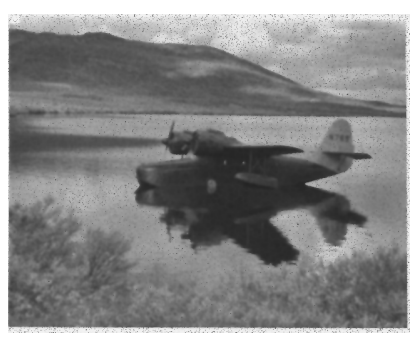

(d) Restored

$\mathrm{PSNR}=25.6970 \mathrm{~dB}, \mathrm{SNR}=11.4072$ VSNR $=16.4865$, SSIM $=0.8497$
Fig. 3. Results of image 3.

TABLE III. PSNR, SNR, VSNR, AND SSIM OF NOISY AND DESPECKLED IMAGE 3 WITH DIFFERENT LEVELS OF $K$

\begin{tabular}{|c|c|c|c|c|}
\hline & PSNR & SNR & VSNR & SSIM \\
\hline $\boldsymbol{K}=\mathbf{4}$ & 10.5052 & -2.6031 & 7.9086 & 0.4547 \\
\hline Proposed & 16.8892 & 5.9958 & 16.0305 & 0.7133 \\
\hline SRAD & 15.3289 & 6.3297 & 15.3680 & 0.7065 \\
\hline $\boldsymbol{K}=\mathbf{1 0}$ & 13.0901 & -0.6873 & 9.5656 & 0.5802 \\
\hline Proposed & 21.6008 & 9.0109 & 16.2655 & 0.7940 \\
\hline SRAD & 18.6167 & 9.4196 & 15.7187 & 0.7807 \\
\hline $\boldsymbol{K}=\mathbf{2 0}$ & 16.5447 & 2.3706 & 12.1235 & 0.7232 \\
\hline Proposed & 25.6970 & 11.4072 & 16.4865 & 0.8497 \\
\hline SRAD & 22.2708 & 11.6397 & 16.0748 & 0.8280 \\
\hline $\boldsymbol{K}=\mathbf{6 0}$ & 28.2317 & 13.7751 & 17.4724 & 0.9661 \\
\hline Proposed & 29.3506 & 14.8891 & 16.9263 & 0.9056 \\
\hline SRAD & 29.2033 & 14.5952 & 15.9009 & 0.8875 \\
\hline
\end{tabular}

\section{CONCLUSION}

In this paper, an efficient approach for image denoising when the image is corrupted by Gamma multiplicative noise was presented. The proposed approach is based on the application of the ENL metric which consists of dividing the image successively in overlapping regions and then calculate automatically the largest value of ENL. The proposed method was applied to three different images and gave better and more satisfactory results than the classical SRAD algorithm. In the future, we plan to work on image denoising in the field of deep learning and compare it with the method presented in the current paper.

\section{ACKNOWLEDGEMENTS}

The authors would like to thank the General Directorate for Scientific Research and Technological Development of the Algerian Republic and the ETA laboratory of Bordj Bou Arreridj University.

\section{REFERENCES}

11] Y. Yu and S. T. Acton, "Speckle reducing anisotropic diffusion," IEEE Transactions on Image Processing, vol. 11, no. 11, pp. 1260-1270, Nov. 2002, https://doi.org/10.1109/TIP.2002.804276.

[2] S. N. Anfinsen, A. P. Doulgeris, and T. Eltoft, "Estimation of the Equivalent Number of Looks in Polarimetric Synthetic Aperture Radar Imagery," IEEE Transactions on Geoscience and Remote Sensing, vol. 47, no. 11, pp. 3795-3809, Nov. 2009, https://doi.org/10.1109/ TGRS.2009.2019269.

[3] K. Kim, S. Jung, and J.-H. Kim, "Adaptive speckle filtering for real-time computing in low earth orbit satellite synthetic aperture radar," ICT Express, vol. 7, no. 2, pp. 187-190, Jun. 2021, https://doi.org/10.1016/ j.icte.2021.02.003.

[4] D. K. Nirmala, "A Brief Study on the Various Noise Models in Digital Image Processing," International Journal of Emerging Technologies in Engineering Research, vol. 5, no. 10, pp. 17-23, Oct. 2017.

[5] N. Diffellah, Z. E. Baarir, F. Derraz, and A. Taleb-Ahmed, "A Global Variational Filter for Restoring Noised Images with Gamma Multiplicative Noise," Engineering, Technology \& Applied Science Research, vol. 9, no. 3, pp. 4188-4195, Jun. 2019, https://doi.org/ 10.48084/etasr.2737.

[6] A. Horé and D. Ziou, "Image Quality Metrics: PSNR vs. SSIM," in 2010 20th International Conference on Pattern Recognition, Istanbul, Turkey, Aug. 2010, pp. 2366-2369, https://doi.org/10.1109/ICPR.2010.579.

[7] M. V. Sarode and P. R. Deshmukh, "Image Sequence Denoising with Motion Estimation in Color Image Sequences," Engineering, Technology \& Applied Science Research, vol. 1, no. 6, pp. 139-143, Dec. 2011 , https://doi.org/10.48084/etasr.54.

[8] H. T. R. Kurmasha, A. F. H. Alharan, C. S. Der, and N. H. Azami, "Enhancement of Edge-based Image Quality Measures Using Entropy for Histogram Equalization-based Contrast Enhancement Techniques," Engineering, Technology \& Applied Science Research, vol. 7, no. 6, pp. 2277-2281, Dec. 2017, https://doi.org/10.48084/etasr.1625.

[9] C. Plapous, C. Marro, and P. Scalart, "Improved Signal-to-Noise Ratio Estimation for Speech Enhancement," IEEE Transactions on Audio, Speech, and Language Processing, vol. 14, no. 6, pp. 2098-2108, Nov. 2006, https://doi.org/10.1109/TASL.2006.872621.

[10] D. M. Chandler and S. S. Hemami, "VSNR: A Wavelet-Based Visual Signal-to-Noise Ratio for Natural Images," IEEE Transactions on Image Processing, vol. 16, no. 9, pp. 2284-2298, Sep. 2007, https://doi.org/10.1109/TIP.2007.901820.

[11] Z. Wang, A. C. Bovik, H. R. Sheikh, and E. P. Simoncelli, "Image quality assessment: from error visibility to structural similarity," IEEE Transactions on Image Processing, vol. 13, no. 4, pp. 600-612, Apr. 2004, https://doi.org/10.1109/TIP.2003.819861.

[12] A. Siddig, Z. Guo, Z. Zhou, and B. Wu, "An image denoising model based on a fourth-order nonlinear partial differential equation," Computers \& Mathematics with Applications, vol. 76, no. 5, pp. 10561074, Sep. 2018, https://doi.org/10.1016/j.camwa.2018.05.040.

[13] R. Chan, H. Yang, and T. Zeng, "A Two-Stage Image Segmentation Method for Blurry Images with Poisson or Multiplicative Gamma 
Noise," SIAM Journal on Imaging Sciences, vol. 7, no. 1, pp. 98-127, Jan. 2014, https://doi.org/10.1137/130920241.

[14] K. Dooley, Image union. 2017.

[15] "Attribution 2.0 Generic - CC BY 2.0," Creative Commons. https://creativecommons.org/licenses/by/2.0/ (accessed Nov. 16, 2021).

[16] NASA Goddard Space Flight Center, First NAC Image Obtained in Mercury Orbit. 2011.

[17] "Transportation airplane on lake." https://free-images.com/display/ transportation_airplane_on_lake.html (accessed Nov. 16, 2021). 